\title{
Ergodic-type method for a system of split variational inclusion and fixed point problems in Hilbert spaces
}

\author{
Dao-Jun Wen*, Yi-An Chen, Ying-Ling Lu \\ College of Mathematics and Statistics, Chongqing Technology and Business University, Chongqing 400067, China. \\ Communicated by Y. H. Yao
}

\begin{abstract}
In this paper, we introduce an ergodic-type method for solving a system of split variational inclusion and fixed point problems of a family of nonexpansive mappings with averaged resolvent operator. We prove that the sequence generated by the proposed algorithm converges strongly to a common element of the set of solutions of a system of split variational inclusion and the set of fixed points of a family of nonexpansive mappings in Hilbert spaces, from which the minimum norm solution is deduced as a special case. Moreover, a numerical example is given to illustrate the operational reliability and convergence of the presented method and results, which may be viewed as a refinement and improvement of the previously known results. (C) 2017 All rights reserved.
\end{abstract}

Keywords: Split variational inclusion, nonexpansive mapping, ergodic-type iteration, fixed point, minimum norm solution. 2010 MSC: 47H10, 41A29, 47J22, 65D15.

\section{Introduction}

Let $\mathrm{H}_{1}$ and $\mathrm{H}_{2}$ be real Hilbert spaces with inner product $\langle. .$.$\rangle and norm \|$.$\| . Recall that a mapping$ $\mathrm{S}: \mathrm{H}_{1} \rightarrow \mathrm{H}_{1}$ is called nonexpansive if

$$
\|S x-S y\| \leqslant\|x-y\|, \quad \forall x, y \in H_{1} .
$$

The fixed point set of $S$ is denoted by Fix(S), i.e., $\operatorname{Fix}(S):=\left\{x \in \mathrm{H}_{1}: S x=x\right\}$.

Recall also that a multi-valued mapping $M: \mathrm{H}_{1} \rightarrow 2^{\mathrm{H}_{1}}$ is called monotone if for all $x, y \in \mathrm{H}_{1}, u \in M x$ and $v \in$ My such that

$$
\langle x-y, u-v\rangle \geqslant 0 .
$$

A monotone mapping $M$ is maximal if the Graph $(M)$ is not properly contained in the graph of any other monotone mapping. Moreover, a monotone mapping $M$ is maximal if and only if for $(x, u) \in$ $\mathrm{H}_{1} \times \mathrm{H}_{1},\langle x-\mathrm{y}, \mathrm{u}-v\rangle \geqslant 0$ for every $(\mathrm{y}, v) \in \operatorname{Graph}(M)$ implies that $u \in M x$.

\footnotetext{
*Corresponding author $\mathrm{Lu})$

Email addresses: daojunwen@163.com (Dao-Jun Wen), chenyian@ctbu.edu.cn (Yi-An Chen), 1376725290@qq.com (Ying-Ling

doi:10.22436/jnsa.010.06.19
}

Received 2017-03-31 
Let $\mathrm{M}: \mathrm{H}_{1} \rightarrow 2^{\mathrm{H}_{1}}$ be a multi-valued maximal monotone mapping. Then the resolvent mapping $\mathrm{J}_{\lambda}^{M}: \mathrm{H}_{1} \rightarrow \mathrm{H}_{1}$ associated with $M$ is defined by

$$
\mathrm{J}_{\lambda}^{M}(x):=(\mathrm{I}+\lambda M)^{-1}(x), \quad \forall x \in \mathrm{H}_{1},
$$

where I stands for the identity operator on $\mathrm{H}_{1}$. It is well-known that for all $\lambda>0$ the resolvent operator $J_{\lambda}^{M}$ is single-valued, nonexpansive and firmly nonexpansive.

We consider the following system of split variational inclusion problem: Find $x^{*} \in \mathrm{H}_{1}$ such that

$$
\left\{\begin{array}{l}
0 \in B\left(x^{*}\right), \\
y_{j}^{*}=A_{j} x^{*} \in H_{2}: 0 \in B_{j}\left(y_{j}^{*}\right), j=1,2, \cdots, m,
\end{array}\right.
$$

where $A_{j}: H_{1} \rightarrow H_{2}$ are bounded linear operators, $B: H_{1} \rightarrow 2^{\mathrm{H}_{1}}$ and $B_{j}: H_{2} \rightarrow 2^{\mathrm{H}_{2}}$ are some multivalued maximal monotone mappings on Hilbert spaces. The set of solution of system (1.1) is denoted by $\mathscr{S}=\left\{x^{*} \in \mathrm{H}_{1}: 0 \in \mathrm{B}\left(\mathrm{x}^{*}\right), \mathrm{y}_{\mathrm{j}}^{*}=\mathrm{A}_{\mathrm{j}} \mathrm{x}^{*} \in \mathrm{H}_{2}: 0 \in \mathrm{B}_{\mathrm{j}}\left(\mathrm{y}_{\mathrm{j}}^{*}\right), \mathrm{j}=1,2, \cdots, \mathrm{m}\right\}$.

Note that, as $\mathfrak{j}=1$, system (1.1) reduces to split variational inclusion problem, which is mainly due to Byrne et al. [3]. The split variational inclusion problem includes split variational inclusion, split fixed point problem, split equilibrium problem, split saddle-point problem and split minimization problem as special cases, which theory and numerical method have been rapidly developed because of its applications in inverse problems, image reconstruction, optimization theory, communication and biomedical engineering; see, for instance, $[2,4-8,10,11,19]$ and the references therein.

In 2014, Kazmi and Rizvi [9] introduced the following iterative method for split variational inclusion and fixed point problem of a nonexpansive mapping:

$$
\left\{\begin{array}{l}
y_{n}=J_{\lambda}^{B}\left[x_{n}-\epsilon A^{*}\left(I-J_{\lambda}^{B_{1}}\right) A x_{n}\right] \\
x_{n+1}=\alpha_{n} f\left(x_{n}\right)+\left(1-\alpha_{n}\right) S y_{n} .
\end{array}\right.
$$

Moreover, they proved that the sequence $\left\{x_{n}\right\}$ generated by (1.2) converges strongly to a common solution of split variational inclusion and fixed point problem of a nonexpansive mapping. In 2015, Wen and Chen [16] and Sitthithakerngkiet et al. [13] extended scheme (1.2) to a general iterative method and a hybrid viscosity algorithm for solving the split variational inclusion in image reconstruction with fixed point problems, respectively.

On the other hand, Shimizu and Takahashi [12] established an ergodic theorem of a family of nonexpansive mappings based on the Cesàro mean. They defined sequence $\left\{x_{n}\right\}$ as follows:

$$
x_{n+1}=\alpha_{n} u+\left(1-\alpha_{n}\right) \frac{1}{n+1} \sum_{i=0}^{n} S^{i} x_{n}
$$

and proved that $\left\{x_{n}\right\}$ converges strongly to a fixed point of $S_{i}$, which is the nearest to $u$.

In 2016, Wang et al. [15] proposed a modified iterative algorithm for a family of split equilibrium problems and fixed problems in Hilbert spaces. They defined $\left\{x_{n}\right\}$ in the following manner:

$$
\left\{\begin{array}{l}
u_{n, j}=T_{r_{n}}^{F}\left[x_{n}-\epsilon A_{j}^{*}\left(I-T_{r_{n}}^{F_{j}}\right) A_{j} x_{n}\right], \quad j=1,2, \cdots, m, \\
y_{n}=P_{C}\left[I-\lambda_{n}\left(\sum_{k=1}^{N} \gamma_{k} B_{k}\right)\right]\left(\frac{1}{m} \sum_{j=1}^{m} u_{n, j}\right), \\
x_{n+1}=\alpha_{n} u+\sum_{i=1}^{n}\left(\alpha_{i-1}-\alpha_{i}\right) S_{i} y_{n},
\end{array}\right.
$$

where $T_{r_{n}}^{F}(x)=\left\{z \in C: F(z, y)+\frac{1}{r_{n}}\langle y-z, z-x\rangle \geqslant 0, \forall y \in C\right\}, P_{C}$ is a metric projection on $C$ and $B_{k}$ is a family of inverse strongly monotone operators. Furthermore, they established a strong convergence theorem for finding a common element of the set of a family of split equilibrium problems and fixed point problems of nonexpansive mappings under certain conditions. 
Inspired and motivated by research going on in this area, we introduce a so-called ergodic-type method for the system of split variational inclusion and fixed point problems of a countable family of nonexpansive mappings via average resolvent operator, which is defined as follows:

$$
\left\{\begin{array}{l}
u_{n, j}=J_{\lambda}^{B}\left[x_{n}-\epsilon A_{j}^{*}\left(I-J_{\lambda}^{B_{j}}\right) A_{j} x_{n}\right], j=1,2, \cdots, m, \\
y_{n}=\beta_{n, 0} x_{n}+\sum_{j=1}^{m} \beta_{n, j} u_{n, j}, \\
x_{n+1}=\alpha_{n} f\left(x_{n}\right)+\sum_{i=1}^{n}\left(\alpha_{i-1}-\alpha_{i}\right) S_{i} y_{n},
\end{array}\right.
$$

where $J_{\lambda}^{B}=(I+\lambda B)^{-1}$, I stands the identity operator on $H_{1}$ and the sequences $\left\{\alpha_{n}\right\},\left\{\beta_{n, j}\right\} \subset[0,1]$ for $j=1,2, \cdots, m$ such that $\sum_{j=0}^{m} \beta_{n, j}=1$.

Our purpose is not only to extend the iterative methods (1.2), (1.3) and (1.4) to the case of the system of split variational inclusion and fixed point problems of a family of nonexpansive mappings in the framework of real Hilbert spaces, but also to establish a strong convergence theorem of the system of split variational inclusion and fixed point problems with variable coefficients instead of mean value, form which the minimum norm solution is deduced as a special case. Moreover, a numerical example is given to illustrate the operational reliability and convergence of our method and results which improve and extend the previously known results of $[9,12,13,15,16]$ and many others.

\section{Preliminaries}

Let $C$ be a nonempty closed convex subset of real Hilbert space $H_{1}$. For every point $x \in H_{1}$, there exists a unique nearest point in $C$, denoted by $P_{C} x$, such that

$$
\left\|x-P_{C} x\right\| \leqslant\|x-y\|, \quad \forall y \in C .
$$

Then $P_{C}$ is called the metric projection of $H_{1}$ onto $C$. It is well-known that $P_{C}$ is nonexpansive and the following inequality holds:

$$
\langle x-u, y-u\rangle \leqslant 0, \quad \forall y \in C,
$$

if and only if $u=P_{C} x$ for given $x \in H_{1}$ and $u \in C$.

Recall that a mapping $\mathrm{f}: \mathrm{H}_{1} \rightarrow \mathrm{H}_{1}$ is called a contraction (or, $\rho$-contraction), if there exists a constant $\rho \in(0,1)$ such that

$$
\|f(x)-f(y)\| \leqslant \rho\|x-y\|, \quad \forall x, y \in H_{1} .
$$

A mapping $\mathrm{T}: \mathrm{H}_{1} \rightarrow \mathrm{H}_{1}$ is called monotone if

$$
\langle T x-T y, x-y\rangle \geqslant 0, \quad \forall x, y \in H_{1} .
$$

T is called strongly monotone if there exists a constant $\alpha>0$ such that

$$
\langle T x-T y, x-y\rangle \geqslant \alpha\|x-y\|^{2}, \quad \forall x, y \in H_{1} .
$$

$\mathrm{T}$ is called $\tau$-inverse strongly monotone (or, $\tau$-ism) if there exists a constant $\tau>0$ such that

$$
\langle T x-T y, x-y\rangle \geqslant \tau\|T x-T y\|^{2}, \quad \forall x, y \in H_{1} .
$$

It is well-known that $I-\lambda T$ is a nonexpansive mapping for each $\lambda \in(0,2 \tau]$ if $T$ is $\tau$-inverse strongly monotone.

In order to prove our main results, we need the following lemmas and results.

Lemma 2.1. Let $\mathrm{H}_{1}$ be a real Hilbert space. The following well-known results hold:

(i) $\|x+y\|^{2} \leqslant\|x\|^{2}+2\langle y,(x+y)\rangle, \quad \forall x, y \in H_{1}$; 
(ii) $\|\mathrm{t} x+(1-\mathrm{t}) \mathrm{y}\|^{2}=\mathrm{t}\|x\|^{2}+(1-\mathrm{t})\|\mathrm{y}\|^{2}-\mathrm{t}(1-\mathrm{t})\|\mathrm{x}-\mathrm{y}\|^{2}, \mathrm{t} \in[0,1], \forall x, y \in \mathrm{H}_{1}$.

Lemma 2.2 ([9]). Split variational inclusion problem (1.1) is equivalent to find $x^{*} \in \mathrm{H}_{1}$ such that $x^{*}=\mathrm{J}_{\lambda}^{\mathrm{B}}\left(\mathrm{x}^{*}\right)$ and $y_{j}^{*}=A_{j} x^{*} \in H_{2}: y_{j}^{*}=J_{\lambda}^{B_{j}}\left(y_{j}^{*}\right)$ for some $\lambda>0$ and $j=1,2, \cdots, m$.

Lemma 2.3 ([14]). A mapping $\mathrm{S}: \mathrm{H}_{1} \rightarrow \mathrm{H}_{1}$ is nonexpansive if and only if the complement $\mathrm{I}-\mathrm{S}$ is $\frac{1}{2}$-inverse strongly monotone.

Lemma 2.4 ([18]). Let $\left\{x_{n}\right\}$ be a bounded sequence in $\mathrm{H}_{1}$ and $\left\{\mathrm{a}_{\mathrm{n}}\right\}$ be a sequence in $[0,1]$ such that $\sum_{n=1}^{\infty} a_{n}=1$. Then we have the following

$$
\left\|\sum_{n=1}^{\infty} a_{n} x_{n}\right\|^{2} \leqslant \sum_{n=1}^{\infty} a_{n}\left\|x_{n}\right\|^{2} .
$$

Recall also that a mapping $\mathrm{V}: \mathrm{H}_{1} \rightarrow \mathrm{H}_{1}$ is said to be averaged if and only if it can be written as the average of the identity mapping and a nonexpansive mapping, i.e., $V:=(1-\alpha) I+\alpha S$, where $\alpha \in(0,1)$ and $\mathrm{S}: \mathrm{H}_{1} \rightarrow \mathrm{H}_{1}$ is a nonexpansive mapping.

Lemma 2.5 ([1]). Let $\mathrm{V}: \mathrm{H}_{1} \rightarrow \mathrm{H}_{1}$ be averaged and $\mathrm{S}: \mathrm{H}_{1} \rightarrow \mathrm{H}_{1}$ be nonexpansive. Then we have

(i) $\mathrm{W}=(1-\alpha) \mathrm{V}+\alpha \mathrm{S}$ is averaged, where $\alpha \in(0,1)$.

(ii) The composite of finitely many averaged mappings is averaged.

(iii) If mappings $\left\{\mathrm{V}_{i}\right\}_{i=1}^{\mathrm{N}}$ are averaged and have a nonempty common fixed point, then

$$
\bigcap_{i=1}^{N} \operatorname{Fix}\left(V_{i}\right)=\operatorname{Fix}\left(V_{1}, V_{2}, \cdots, V_{N}\right)
$$

Obviously, averaged mappings are nonexpansive. Further, firmly nonexpansive mappings (in particular, projections on nonempty closed and convex subsets and resolvent operators of maximal monotone operators) are averaged.

Lemma 2.6 ([17]). Let $\left\{a_{n}\right\}_{n=1}^{\infty}$ be a sequence of nonnegative real numbers such that

$$
a_{n+1} \leqslant\left(1-\tau_{n}\right) a_{n}+\delta_{n}, \quad n \geqslant 1,
$$

where $\left\{\tau_{\mathfrak{n}}\right\}_{\mathfrak{n}=1}^{\infty}$ is a sequence in $(0,1)$ and $\left\{\delta_{\mathfrak{n}}\right\}_{\mathfrak{n}=1}^{\infty}$ is a sequence such that

(i) $\sum_{n=1}^{\infty} \tau_{n}=\infty$;

(ii) $\limsup _{n \rightarrow \infty} \frac{\delta_{n}}{\tau_{n}} \leqslant 0$, or $\sum_{n=1}^{\infty}\left|\delta_{n}\right|<\infty$.

Then $\lim _{n \rightarrow \infty} a_{n}=0$.

\section{Main results}

Theorem 3.1. Let $\mathrm{H}_{1}$ and $\mathrm{H}_{2}$ be two real Hilbert spaces. Let $\mathrm{B}: \mathrm{H}_{1} \rightarrow 2^{\mathrm{H}_{1}}, \mathrm{~B}_{\mathrm{j}}: \mathrm{H}_{2} \rightarrow 2^{\mathrm{H}_{2}}$ be some maximal monotone mappings and $\mathrm{A}_{\mathrm{j}}: \mathrm{H}_{1} \rightarrow \mathrm{H}_{2}$ be a family of bounded linear operators for $\mathrm{j}=1,2, \cdots, \mathrm{m}$. Let $\mathrm{f}$ be a $\rho$ contraction and $\left\{\mathrm{S}_{\mathrm{n}}\right\}$ be a countable family of nonexpansive mappings on $\mathrm{H}_{1}$ such that $\Omega=\bigcap_{\mathrm{n}=1}^{\infty}$ Fix $\left(\mathrm{S}_{\mathrm{n}}\right) \cap \mathscr{S} \neq$ $\emptyset$. Assume that $\alpha_{0}=1$ and $\left\{\alpha_{n}\right\}_{\mathfrak{n}=1}^{\infty}$ is a strictly decreasing sequence in $[0,1]$. For given $x_{1} \in H_{1},\left\{\beta_{\mathfrak{n}, j}\right\}_{\mathfrak{n}=1}^{\infty} \subset[0,1]$ and the following conditions are satisfied:

(i) $\lim _{n \rightarrow \infty} \alpha_{n}=0, \sum_{n=1}^{\infty} \alpha_{n}=\infty$ and $\sum_{n=1}^{\infty}\left|\alpha_{n-1}-\alpha_{n}\right|<\infty$;

(ii) $\sum_{j=0}^{m} \beta_{n, j}=1, \liminf _{n \rightarrow \infty} \beta_{n, j}>0$ and $\sum_{n=1}^{\infty}\left|\beta_{n, j}-\beta_{n-1, j}\right|<\infty$, for all $j=0,1,2, \cdots$, m. 
If $\lambda \in(0,1)$ and $\epsilon \in\left(0, \frac{1}{L}\right)$, where $L=\max _{1 \leqslant j \leqslant m}\left\{L_{j}\right\}, L_{j}$ is the spectral radius of the operator $A_{j}^{*} A_{j}$ and $A_{j}^{*}$ is the adjoint of $A_{j}$, then the sequence $\left\{x_{n}\right\}$ generated by (1.5) converges strongly to $q \in \Omega$, which is the unique solution of the variational inequality:

$$
\langle\mathrm{f}(\mathrm{q})-\mathrm{q}, w-\mathrm{q}\rangle \leqslant 0, \quad \forall w \in \Omega .
$$

Proof. Taking $p \in \Omega$, we have $p=J_{\lambda}^{B} p, A_{j} p=J_{\lambda}^{B_{j}} A_{j} p$, for $j=1,2, \cdots, m$ and $S_{i} p=p$ for $i=1,2, \cdots$. Since $J_{\lambda}^{B}$ and $J_{\lambda}^{B_{j}}$ are firmly nonexpansive, they are averaged and hence nonexpansive. For $\in \in\left(0, \frac{1}{L}\right)$, the mappings $\left[I-\epsilon A_{j}^{*}\left(I-J_{\lambda}^{B_{j}}\right) A_{j}\right]$ are averaged, see $[9,10]$. From $(1.5)$, we have

$$
\begin{aligned}
\left\|u_{n, j}-p\right\| & =\left\|J_{\lambda}^{B}\left[x_{n}-\epsilon A_{j}^{*}\left(I-J_{\lambda}^{B_{j}}\right) A_{j} x_{n}\right]-J_{\lambda}^{B} p\right\| \\
& \leqslant\left\|\left[I-\epsilon A_{j}^{*}\left(I-J_{\lambda}^{B_{j}}\right) A_{j}\right] x_{n}-p\right\| \\
& \leqslant\left\|x_{n}-p\right\| .
\end{aligned}
$$

By $\beta_{n, 0}+\sum_{j=1}^{m} \beta_{n, j}=1$ and (3.1), we obtain

$$
\begin{aligned}
\left\|y_{n}-p\right\| & =\left\|\beta_{n, 0}\left(x_{n}-p\right)+\sum_{j=1}^{m} \beta_{n, j}\left(u_{n, j}-p\right)\right\| \\
& \leqslant \beta_{n, 0}\left\|x_{n}-p\right\|+\sum_{j=1}^{m} \beta_{n, j}\left\|u_{n, j}-p\right\| \\
& \leqslant\left\|x_{n}-p\right\| .
\end{aligned}
$$

From (1.5) again, we have

$$
\begin{aligned}
\left\|x_{n+1}-p\right\| & =\left\|\alpha_{n} f\left(x_{n}\right)+\sum_{i=1}^{n}\left(\alpha_{i-1}-\alpha_{i}\right) S_{i} y_{n}-p\right\| \\
& \leqslant \alpha_{n}\left\|f\left(x_{n}\right)-p\right\|+\sum_{i=1}^{n}\left(\alpha_{i-1}-\alpha_{i}\right)\left\|S_{i} y_{n}-p\right\| \\
& \leqslant \alpha_{n}\left\|f\left(x_{n}\right)-f(p)\right\|+\alpha_{n}\|f(p)-p\|+\sum_{i=1}^{n}\left(\alpha_{i-1}-\alpha_{i}\right)\left\|y_{n}-p\right\| \\
& \leqslant \alpha_{n} \rho\left\|x_{n}-p\right\|+\alpha_{n}\|f(p)-p\|+\sum_{i=1}^{n}\left(\alpha_{i-1}-\alpha_{i}\right)\left\|x_{n}-p\right\| \\
& =\left[1-(1-\rho) \alpha_{n}\right]\left\|x_{n}-p\right\|+\alpha_{n}\|f(p)-p\| .
\end{aligned}
$$

By a simple induction, we estimate

$$
\left\|x_{n}-p\right\| \leqslant \max \left\{\left\|x_{1}-p\right\|, \frac{1}{1-\rho}\|f(p)-p\|\right\} .
$$

Therefore, sequence $\left\{x_{n}\right\}$ is bounded, and so are sequences $\left\{y_{n}\right\},\left\{u_{n, j}\right\},\left\{f\left(x_{n}\right)\right\}$ and $\left\{S_{n} y_{n}\right\}$.

Next, we show that $\lim _{n \rightarrow \infty}\left\|x_{n+1}-x_{n}\right\|=0$. Note that $J_{\lambda}^{B}\left[I-\epsilon A_{j}^{*}\left(I-J_{\lambda}^{B_{j}}\right) A_{j}\right]$ is averaged and nonexpansive by Lemma 2.5. Using (1.5), we have

$$
\begin{aligned}
\left\|u_{n, j}-u_{n-1, j}\right\| & =\left\|J_{\lambda}^{B}\left[x_{n}-\epsilon A_{j}^{*}\left(I-J_{\lambda}^{B_{j}}\right) A_{j} x_{n}\right]-J_{\lambda}^{B}\left[x_{n-1}-\epsilon A_{j}^{*}\left(I-J_{\lambda}^{B_{j}}\right) A_{j} x_{n-1}\right]\right\| \\
& \leqslant\left\|x_{n}-x_{n-1}\right\| .
\end{aligned}
$$


Moreover, we have

$$
\begin{aligned}
\left\|y_{n}-y_{n-1}\right\| & =\left\|\beta_{n, 0} x_{n}+\sum_{j=1}^{m} \beta_{n, j} u_{n, j}-\beta_{n-1,0} x_{n-1}-\sum_{j=1}^{m} \beta_{n-1, j} u_{n-1, j}\right\| \\
& \leqslant\left\|\beta_{n, 0} x_{n}-\beta_{n-1,0} x_{n-1}\right\|+\sum_{j=1}^{m}\left\|\beta_{n, j} u_{n, j}-\beta_{n-1, j} u_{n-1, j}\right\| \\
& \leqslant\left\|\beta_{n, 0} x_{n}-\beta_{n-1,0} x_{n-1}\right\|+\sum_{j=1}^{m} \beta_{n, j}\left\|u_{n, j}-u_{n-1, j}\right\|+\sum_{j=1}^{m}\left|\beta_{n, j}-\beta_{n-1, j}\right| \mid u_{n-1, j} \| \\
& \leqslant\left\|x_{n}-x_{n-1}\right\|+\left|\beta_{n, 0}-\beta_{n-1,0}\right|\left\|x_{n-1}\right\|+\sum_{j=1}^{m}\left|\beta_{n, j}-\beta_{n-1, j}\right|\left\|u_{n-1, j}\right\| .
\end{aligned}
$$

Since $S_{i}$ is nonexpansive, we further obtain

$$
\begin{aligned}
\left\|x_{n+1}-x_{n}\right\|= & \left\|\alpha_{n} f\left(x_{n}\right)+\sum_{i=1}^{n}\left(\alpha_{i-1}-\alpha_{i}\right) S_{i} y_{n}-\alpha_{n-1} f\left(x_{n-1}\right)-\sum_{i=1}^{n-1}\left(\alpha_{i-1}-\alpha_{i}\right) S_{i} y_{n-1}\right\| \\
\leqslant & \left\|\alpha_{n} f\left(x_{n}\right)-\alpha_{n-1} f\left(x_{n-1}\right)\right\|+\sum_{i=1}^{n-1}\left(\alpha_{i-1}-\alpha_{i}\right)\left\|S_{i} y_{n}-S_{i} y_{n-1}\right\|+\left(\alpha_{n-1}-\alpha_{n}\right)\left\|S_{n} y_{n}\right\| \\
\leqslant & \left\|\alpha_{n} f\left(x_{n}\right)-\alpha_{n-1} f\left(x_{n-1}\right)\right\|+\sum_{i=1}^{n-1}\left(\alpha_{i-1}-\alpha_{i}\right)\left\|y_{n}-y_{n-1}\right\|+\left(\alpha_{n-1}-\alpha_{n}\right)\left\|S_{n} y_{n}\right\| \\
\leqslant & \left|\alpha_{n}-\alpha_{n-1}\right|\left\|f\left(x_{n}\right)\right\|+\alpha_{n-1} \rho\left\|x_{n}-x_{n-1}\right\|+\left(1-\alpha_{n-1}\right)\left\|x_{n}-x_{n-1}\right\| \\
& +\left|\beta_{n, 0}-\beta_{n-1,0}\right|\left\|x_{n-1}\right\|+\sum_{j=1}^{m}\left|\beta_{n, j}-\beta_{n-1, j}\right|\left\|u_{n-1, j}\right\|+\left(\alpha_{n-1}-\alpha_{n}\right)\left\|S_{n} y_{n}\right\| \\
\leqslant & {\left[1-(1-\rho) \alpha_{n-1}\right]\left\|x_{n}-x_{n-1}\right\|+\left(\alpha_{n-1}-\alpha_{n}\right) M_{1}+\sum_{j=0}^{m}\left|\beta_{n, j}-\beta_{n-1, j}\right| M_{2} }
\end{aligned}
$$

where $M_{1}=\max \left\{\left\|f\left(x_{n}\right)\right\|,\left\|S_{n} y_{n}\right\|\right\}$ and $M_{2}=\max \left\{\left\|x_{n-1}\right\|, \sup _{1 \leqslant j \leqslant m}\left\|u_{n-1, j}\right\|\right\}$. It follows from (3.3) and Lemma 2.6 that

$$
\lim _{n \rightarrow \infty}\left\|x_{n+1}-x_{n}\right\|=0
$$

Now, we prove that $\lim _{n \rightarrow \infty}\left\|S_{i} x_{n}-x_{n}\right\|=0$ for $i=1,2, \cdots$. To do this, we first prove that $A_{j}^{*}(I-$ $\left.J_{\lambda}^{B_{j}}\right) A_{j}$ is a $\frac{1}{2 L_{j}}$-inverse strong monotone. It follows from Lemma 2.3 that

$$
\begin{aligned}
\Theta & =\left\|A_{j}^{*}\left(I-J_{\lambda}^{B_{j}}\right) A_{j} x-A_{j}^{*}\left(I-J_{\lambda}^{B_{j}}\right) A_{j} y\right\|^{2} \\
& =\left\langle\left(I-J_{\lambda}^{B_{j}}\right)\left(A_{j} x-A_{j} y\right), A_{j} A_{j}^{*}\left(I-J_{\lambda}^{B_{j}}\right)\left(A_{j} x-A_{j} y\right)\right\rangle \\
& \leqslant L_{j}\left\langle\left(I-J_{\lambda}^{B_{j}}\right)\left(A_{j} x-A_{j} y\right),\left(I-J_{\lambda}^{B_{j}}\right)\left(A_{j} x-A_{j} y\right)\right\rangle \\
& \leqslant L_{j}\left\|\left(I-J_{\lambda}^{B_{j}}\right)\left(A_{j} x-A_{j} y\right)\right\|^{2} \\
& \leqslant 2 L_{j}\left\langle A_{j} x-A_{j} y,\left(I-J_{\lambda}^{B_{j}}\right)\left(A_{j} x-A_{j} y\right)\right\rangle \\
& =2 L_{j}\left\langle x-y, A_{j}^{*}\left(I-J_{\lambda}^{B_{j}}\right) A_{j}(x-y)\right\rangle,
\end{aligned}
$$

for all $x, y \in H_{1}$, which implies that $A_{j}^{*}\left(I-J_{\lambda}^{B_{j}}\right) A_{j}$ is a $\frac{1}{2 L_{j}}$-inverse strong monotone. Since $J_{\lambda}^{B}$ is firmly nonexpansive and

$$
\left\|u_{n, j}-p\right\|^{2}=\left\|J_{\lambda}^{B}\left[I-\epsilon A_{j}^{*}\left(I-J_{\lambda}^{B_{j}}\right) A_{j}\right] x_{n}-J_{\lambda}^{B} p\right\|^{2}
$$




$$
\begin{aligned}
& \leqslant\left\|x_{n}-p-\epsilon\left[A_{j}^{*}\left(I-J_{\lambda}^{B_{j}}\right) A_{j} x_{n}-A_{j}^{*}\left(I-J_{\lambda}^{B_{j}}\right) A_{j} p\right]\right\|^{2} \\
& =\left\|x_{n}-p\right\|^{2}-2 \epsilon\left\langle x_{n}-p, A_{j}^{*}\left(I-J_{\lambda}^{B_{j}}\right) A_{j}\left(x_{n}-p\right)\right\rangle+\epsilon^{2}\left\|A_{j}^{*}\left(I-J_{\lambda}^{B_{j}}\right) A_{j}\left(x_{n}-p\right)\right\|^{2} \\
& \leqslant\left\|x_{n}-p\right\|^{2}+\epsilon\left(\epsilon-\frac{1}{L_{j}}\right)\left\|A_{j}^{*}\left(I-J_{\lambda}^{B_{j}}\right) A_{j} x_{n}-A_{j}^{*}\left(I-J_{\lambda}^{B_{j}}\right) A_{j} p\right\|^{2} \\
& =\left\|x_{n}-p\right\|^{2}+\epsilon\left(\epsilon-\frac{1}{L_{j}}\right)\left\|A_{j}^{*}\left(I-J_{\lambda}^{B_{j}}\right) A_{j} x_{n}\right\|^{2} .
\end{aligned}
$$

By (3.5) and Lemma 2.4, we have

$$
\begin{aligned}
\left\|y_{n}-p\right\|^{2} & =\left\|\beta_{n, 0}\left(x_{n}-p\right)+\sum_{j=1}^{m} \beta_{n, j}\left(u_{n, j}-p\right)\right\|^{2} \\
& \leqslant \beta_{n, 0}\left\|x_{n}-p\right\|^{2}+\sum_{j=1}^{m} \beta_{n, j}\left\|u_{n, j}-p\right\|^{2} \\
& \leqslant\left\|x_{n}-p\right\|^{2}+\sum_{j=1}^{m} \beta_{n, j} \epsilon\left(\epsilon-\frac{1}{L_{j}}\right)\left\|A_{j}^{*}\left(I-J_{\lambda}^{B_{j}}\right) A_{j} x_{n}\right\|^{2} .
\end{aligned}
$$

From (1.5) and (3.6), we obtain

$$
\begin{aligned}
\left\|x_{n+1}-p\right\|^{2} & =\left\|\alpha_{n} f\left(x_{n}\right)+\sum_{i=1}^{n}\left(\alpha_{i-1}-\alpha_{i}\right) S_{i} y_{n}-p\right\|^{2} \\
& \leqslant \alpha_{n}\left\|f\left(x_{n}\right)-p\right\|^{2}+\sum_{i=1}^{n}\left(\alpha_{i-1}-\alpha_{i}\right)\left\|S_{i} y_{n}-p\right\|^{2} \\
& \leqslant \alpha_{n}\left\|f\left(x_{n}\right)-p\right\|^{2}+\sum_{i=1}^{n}\left(\alpha_{i-1}-\alpha_{i}\right)\left\|y_{n}-p\right\|^{2} \\
& \leqslant \alpha_{n}\left\|f\left(x_{n}\right)-p\right\|^{2}+\left\|x_{n}-p\right\|^{2}+\sum_{j=1}^{m} \beta_{n, j} \epsilon\left(\epsilon-\frac{1}{L_{j}}\right)\left\|A_{j}^{*}\left(I-J_{\lambda}^{B_{j}}\right) A_{j} x_{n}\right\|^{2},
\end{aligned}
$$

which implies that

$$
\begin{aligned}
\beta_{n, j} \epsilon\left(\epsilon-\frac{1}{L_{j}}\right)\left\|A_{j}^{*}\left(I-J_{\lambda}^{B_{j}}\right) A_{j} x_{n}\right\|^{2} & \leqslant \sum_{j=1}^{m} \beta_{n, j} \epsilon\left(\epsilon-\frac{1}{L_{j}}\right)\left\|A_{j}^{*}\left(I-J_{\lambda}^{B_{j}}\right) A_{j} x_{n}\right\|^{2} \\
& \leqslant \alpha_{n}\left\|f\left(x_{n}\right)-p\right\|^{2}+\left\|x_{n}-p\right\|^{2}-\left\|x_{n+1}-p\right\|^{2} \\
& \leqslant \alpha_{n}\left\|f\left(x_{n}\right)-p\right\|^{2}+\left\|x_{n+1}-x_{n}\right\|\left(\left\|x_{n}-p\right\|+\left\|x_{n+1}-p\right\|\right) .
\end{aligned}
$$

It follows from condition (i) and (3.4) that

$$
\lim _{n \rightarrow \infty}\left\|\left(I-J_{\lambda}^{B_{j}}\right) A_{j} x_{n}\right\|=0, \quad j=1,2, \cdots, m .
$$

Since $J_{\lambda}^{B}$ is firmly nonexpansive and $I-\epsilon A_{j}^{*}\left(I-J_{\lambda}^{B_{j}}\right) A_{j}$ is nonexpansive, we have

$$
\begin{aligned}
\left\|u_{n, j}-p\right\|^{2} & =\left\|J_{\lambda}^{B}\left[x_{n}-\epsilon A_{j}^{*}\left(I-J_{\lambda}^{B_{j}}\right) A_{j} x_{n}\right]-J_{\lambda}^{B} p\right\|^{2} \\
& \leqslant\left\langle u_{n, j}-p, x_{n}-\epsilon A_{j}^{*}\left(I-J_{\lambda}^{B_{j}}\right) A_{j} x_{n}-p\right\rangle \\
& =\frac{1}{2}\left\{\left\|u_{n, j}-p\right\|^{2}+\left\|x_{n}-\epsilon A_{j}^{*}\left(I-J_{\lambda}^{B_{j}}\right) A_{j} x_{n}-p\right\|^{2}-\left\|u_{n, j}-x_{n}+\epsilon A_{j}^{*}\left(I-J_{\lambda}^{B_{j}}\right) A_{j} x_{n}\right\|^{2}\right\}
\end{aligned}
$$




$$
\begin{aligned}
\leqslant & \frac{1}{2}\left\{\left\|u_{n, j}-p\right\|^{2}+\left\|x_{n}-p\right\|^{2}-\left\|u_{n, j}-x_{n}\right\|^{2}-\epsilon^{2}\left\|A_{j}^{*}\left(I-J_{\lambda}^{B_{j}}\right) A_{j} x_{n}\right\|^{2}\right. \\
& \left.-2 \epsilon\left\langle u_{n, j}-x_{n}, A_{j}^{*}\left(I-J_{\lambda}^{B_{j}}\right) A_{j} x_{n}\right\rangle\right\} .
\end{aligned}
$$

Thus, we deduce that

$$
\left\|u_{n, j}-p\right\|^{2} \leqslant\left\|x_{n}-p\right\|^{2}-\left\|u_{n, j}-x_{n}\right\|^{2}+2 \epsilon\left\|u_{n, j}-x_{n}\right\|\left\|A_{j}^{*}\left(I-J_{\lambda}^{B_{j}}\right) A_{j} x_{n}\right\| .
$$

Combining (3.6), (3.7) and (3.9), we have

$$
\begin{aligned}
\left\|x_{n+1}-p\right\|^{2} \leqslant & \alpha_{n}\left\|f\left(x_{n}\right)-p\right\|^{2}+\left(1-\alpha_{n}\right)\left\|y_{n}-p\right\|^{2} \\
\leqslant & \alpha_{n}\left\|f\left(x_{n}\right)-p\right\|^{2}+\beta_{n, 0}\left\|x_{n}-p\right\|^{2}+\sum_{j=1}^{m} \beta_{n, j}\left\|u_{n, j}-p\right\|^{2} \\
\leqslant & \alpha_{n}\left\|f\left(x_{n}\right)-p\right\|^{2}+\left\|x_{n}-p\right\|^{2}-\sum_{j=1}^{m} \beta_{n, j}\left\|u_{n, j}-x_{n}\right\|^{2} \\
& +2 \epsilon \sum_{j=1}^{m} \beta_{n, j}\left\|u_{n, j}-x_{n}\right\|\left\|A_{j}^{*}\left(I-J_{\lambda}^{B_{j}}\right) A_{j} x_{n}\right\|,
\end{aligned}
$$

which implies that

$$
\begin{aligned}
\beta_{n, j}\left\|u_{n, j}-x_{n}\right\|^{2} \leqslant & \sum_{j=1}^{m} \beta_{n, j}\left\|u_{n, j}-x_{n}\right\|^{2} \\
\leqslant & \alpha_{n}\left\|f\left(x_{n}\right)-p\right\|^{2}+\left\|x_{n+1}-x_{n}\right\|\left(\left\|x_{n+1}-p\right\|+\left\|x_{n}-p\right\|\right) \\
& +2 \epsilon \sum_{j=1}^{m} \beta_{n, j}\left\|u_{n, j}-x_{n}\right\|\left\|A_{j}^{*}\left(I-J_{\lambda}^{B_{j}}\right) A_{j} x_{n}\right\| .
\end{aligned}
$$

Together with condition (i), (3.4) and (3.8), we arrive at

$$
\lim _{n \rightarrow \infty}\left\|u_{n, j}-x_{n}\right\|=0, \quad j=1,2, \cdots, m .
$$

Moreover, by (1.5), $y_{n}-x_{n}=\sum_{j=1}^{m} \beta_{n, j}\left(u_{n, j}-x_{n}\right)$, we have

$$
\lim _{n \rightarrow \infty}\left\|y_{n}-x_{n}\right\|=0
$$

From (1.5) again, we obtain

$$
x_{n+1}-y_{n}=\alpha_{n}\left[f\left(x_{n}\right)-y_{n}\right]+\sum_{i=1}^{n}\left(\alpha_{i-1}-\alpha_{i}\right)\left(S_{i} y_{n}-y_{n}\right)
$$

Since $\left\{\alpha_{n}\right\}_{n=1}^{\infty}$ is a strictly decreasing sequence, we find that

$$
\begin{aligned}
\left(\alpha_{i-1}-\alpha_{i}\right)\left\|S_{i} y_{n}-y_{n}\right\|^{2} & \leqslant \sum_{j=1}^{n}\left(\alpha_{i-1}-\alpha_{i}\right)\left\|S_{i} y_{n}-y_{n}\right\|^{2} \\
& \leqslant 2 \sum_{j=1}^{n}\left(\alpha_{i-1}-\alpha_{i}\right)\left\langle S_{i} y_{n}-y_{n}, p-y_{n}\right\rangle \\
& \leqslant 2\left\langle x_{n+1}-y_{n}, p-y_{n}\right\rangle-2 \alpha_{n}\left\langle f\left(x_{n}\right)-y_{n}, p-y_{n}\right\rangle \\
& \leqslant 2\left\|x_{n+1}-y_{n}\right\|\left\|p-y_{n}\right\|+2 \alpha_{n}\left\|f\left(x_{n}\right)-y_{n}\right\|\left\|y_{n}-p\right\|
\end{aligned}
$$




$$
\leqslant 2\left(\left\|x_{n+1}-x_{n}\right\|+\left\|x_{n}-y_{n}\right\|\right)\left\|p-y_{n}\right\|+2 \alpha_{n}\left\|f\left(x_{n}\right)-y_{n}\right\|\left\|y_{n}-p\right\| .
$$

By (3.4), (3.11) and condition (i), we have

$$
\lim _{n \rightarrow \infty}\left\|S_{i} y_{n}-y_{n}\right\|=0, \quad i=1,2, \cdots
$$

Note that

$$
\begin{aligned}
\left\|S_{i} x_{n}-x_{n}\right\| & \leqslant\left\|S_{i} x_{n}-S_{i} y_{n}\right\|+\left\|S_{i} y_{n}-y_{n}\right\|+\left\|y_{n}-x_{n}\right\| \\
& \leqslant 2\left\|x_{n}-y_{n}\right\|+\left\|S_{i} y_{n}-y_{n}\right\| .
\end{aligned}
$$

It follows from (3.11) and (3.12) that

$$
\lim _{n \rightarrow \infty}\left\|S_{i} x_{n}-x_{n}\right\|=0, \quad i=1,2, \cdots
$$

Since $\left\{x_{n}\right\}$ is bounded, without loss of generality, we assume that $\left\{x_{n_{k}}\right\}$ is a subsequence of $\left\{x_{n}\right\}$ such that $\left\{x_{n_{k}}\right\}$ converges weakly to $w$, i.e., $x_{n_{k}} \rightarrow w$ as $k \rightarrow \infty$. We claim that $w \in \operatorname{Fix}\left(S_{i}\right)$. Indeed, assume that $w \neq S_{i} w$, we have

$$
\begin{aligned}
\liminf _{k \rightarrow \infty}\left\|x_{n_{k}}-w\right\| & <\liminf _{k \rightarrow \infty}\left\|x_{n_{k}}-S_{i} w\right\| \\
& \leqslant \liminf _{k \rightarrow \infty}\left\{\left\|x_{n_{k}}-S_{i} x_{n_{k}}\right\|+\left\|S_{i} x_{n_{k}}-S_{i} w\right\|\right\} \\
& \leqslant \liminf _{k \rightarrow \infty}\left\|x_{n_{k}}-w\right\|,
\end{aligned}
$$

which is a contradiction arising from Opial's condition. Therefore, we obtain $w \in \operatorname{Fix}\left(S_{i}\right)$. On the other hand, $u_{n_{k}, j}=J_{\lambda}^{B}\left[x_{n_{k}}-\epsilon A_{j}^{*}\left(I-J_{\lambda}^{B j}\right) A_{j} x_{n_{k}}\right]$ can be rewritten as

$$
\frac{\left(x_{n_{k}}-u_{n_{k}, j}\right)-\epsilon A_{j}^{*}\left(I-J_{\lambda}^{B}\right) A_{j} x_{n_{k}}}{\lambda} \in B u_{n_{k}, j}, j=1,2, \cdots, m .
$$

Taking limit $k \rightarrow \infty$ in (3.13) and by using (3.8), (3.10) and the fact that the graph of a maximal monotone operator is weakly-strongly closed, we can obtain $0 \in \mathrm{B}(w)$. Moreover, since $\left\{x_{n}\right\}$ and $\left\{u_{n, j}\right\}$ have the same asymptotical behavior, $\left\{A_{j} x_{n_{k}}\right\}$ weakly converges to $A_{j} w$. By the fact that $J_{\lambda}^{B_{j}}$ is nonexpansive and (3.8), we obtain $0 \in B_{j}(A w)$ for $j=1,2, \cdots, m$. It follows from Lemma 2.2 that $w \in \mathscr{S}$. Consequently, $w \in \Omega=\bigcap_{\mathrm{n}=1}^{\infty} \operatorname{Fix}\left(S_{\mathrm{n}}\right) \bigcap \mathscr{S}$.

Finally, we prove that $\left\{x_{n}\right\}$ converges strongly to $q$, where $q=P_{\Omega} f(q)$. Note that the subsequence $\left\{x_{n_{k}}\right\}$ of $\left\{x_{n}\right\}$ converges weakly to $w$ and

$$
\limsup _{n \rightarrow \infty}\left\langle f(q)-q, x_{n}-q\right\rangle=\lim _{k \rightarrow \infty}\left\langle f(q)-q, x_{n_{k}}-q\right\rangle=\langle f(q)-q, w-q\rangle \leqslant 0 .
$$

In addition, we show that the uniqueness of a solution of the variational inequality

$$
\langle f(x)-x, w-x\rangle \leqslant 0, \quad \forall w \in \Omega .
$$

Suppose $\mathrm{q} \in \Omega$ and $\hat{\mathrm{q}} \in \Omega$ are solutions to (3.15), then

$$
\langle f(q)-q, \hat{q}-q\rangle \leqslant 0,
$$

and

$$
\langle f(\hat{q})-\hat{q}, q-\hat{q}\rangle \leqslant 0 .
$$


Adding up (3.16) and (3.17) one gets

$$
\langle q-f(q)-(\hat{q}-f(\hat{q})), q-\hat{q}\rangle \leqslant 0,
$$

which implies that

$$
\rho\|q-\hat{q}\|^{2} \geqslant\langle f(q)-f(\hat{q}), q-\hat{q}\rangle \geqslant\langle q-\hat{q}, q-\hat{q}\rangle=\|q-\hat{q}\|^{2} .
$$

Thus from $\rho \in[0,1)$, it follows that $q=\hat{q}$, the uniqueness is proved. Furthermore, by (1.5) and (3.2), we obtain

$$
\begin{aligned}
\left\|x_{n+1}-q\right\|^{2}= & \alpha_{n}\left\langle f\left(x_{n}\right)-q, x_{n+1}-q\right\rangle+\sum_{i=1}^{n}\left(\alpha_{i-1}-\alpha_{i}\right)\left\langle S_{i} y_{n}-q, x_{n+1}-q\right\rangle \\
\leqslant & \alpha_{n}\left\langle f\left(x_{n}\right)-q, x_{n+1}-q\right\rangle+\frac{1}{2} \sum_{i=1}^{n}\left(\alpha_{i-1}-\alpha_{i}\right)\left(\left\|S_{i} y_{n}-q\right\|^{2}+\left\|x_{n+1}-q\right\|^{2}\right) \\
\leqslant & \alpha_{n}\left\langle f\left(x_{n}\right)-q, x_{n+1}-q\right\rangle+\frac{1}{2} \sum_{i=1}^{n}\left(\alpha_{i-1}-\alpha_{i}\right)\left(\left\|y_{n}-q\right\|^{2}+\left\|x_{n+1}-q\right\|^{2}\right) \\
\leqslant & \alpha_{n}\left\langle f\left(x_{n}\right)-f(q), x_{n+1}-q\right\rangle+\alpha_{n}\left\langle f(q)-q, x_{n+1}-q\right\rangle \\
& +\frac{1}{2}\left(1-\alpha_{n}\right)\left(\left\|x_{n}-q\right\|^{2}+\left\|x_{n+1}-q\right\|^{2}\right) \\
\leqslant & \frac{1}{2} \alpha_{n}\left(\left\|f\left(x_{n}\right)-f(q)\right\|^{2}+\left\|x_{n+1}-q\right\|^{2}\right)+\alpha_{n}\left\langle f(q)-q, x_{n+1}-q\right\rangle \\
& +\frac{1}{2}\left(1-\alpha_{n}\right)\left(\left\|x_{n}-q\right\|^{2}+\left\|x_{n+1}-q\right\|^{2}\right) \\
\leqslant & \frac{1}{2}\left[1-(1-\rho) \alpha_{n}\right]\left\|x_{n}-q\right\|^{2}+\frac{1}{2}\left\|x_{n+1}-q\right\|^{2}+\alpha_{n}\left\langle f(q)-q, x_{n+1}-q\right\rangle .
\end{aligned}
$$

This implies that

$$
\left\|x_{n+1}-q\right\|^{2} \leqslant\left[1-(1-\rho) \alpha_{n}\right]\left\|x_{n}-q\right\|^{2}+2 \alpha_{n}\left\langle f(q)-q, x_{n+1}-q\right\rangle .
$$

From the condition (i), (3.14) and Lemma 2.6, we obtain the desired conclusion that $\left\{x_{n}\right\}$ converges strongly to $\mathrm{q} \in \Omega$. This completes the proof.

Theorem 3.2. Let $\mathrm{H}_{1}$ and $\mathrm{H}_{2}$ be two real Hilbert spaces. Let $\mathrm{B}: \mathrm{H}_{1} \rightarrow 2^{\mathrm{H}_{1}}, \mathrm{~B}_{\mathrm{j}}: \mathrm{H}_{2} \rightarrow 2^{\mathrm{H}_{2}}$ be some maximal monotone mappings and $A_{j}: \mathrm{H}_{1} \rightarrow \mathrm{H}_{2}$ be a family of bounded linear operators for $j=1,2, \cdots, \mathrm{m}$. Let $\left\{\mathrm{S}_{\mathrm{n}}\right\}$ be a countable family of nonexpansive mappings on $\mathrm{H}_{1}$ such that $\Omega=\bigcap_{\mathrm{n}=1}^{\infty} \operatorname{Fix}\left(\mathrm{S}_{\mathrm{n}}\right) \cap \mathscr{S} \neq \emptyset$. For given $\mathrm{x}_{1} \in \mathrm{H}_{1}$ and $\lambda \in(0,1)$, define $\left\{x_{n}\right\}$ in the following manner:

$$
\left\{\begin{array}{l}
u_{n, j}=J_{\lambda}^{B}\left[x_{n}-\epsilon A_{j}^{*}\left(I-J_{\lambda}^{B_{j}}\right) A_{j} x_{n}\right], j=1,2, \cdots, m, \\
y_{n}=\beta_{n, 0} x_{n}+\sum_{j=1}^{m} \beta_{n, j} u_{n, j}, \\
x_{n+1}=\sum_{i=1}^{n}\left(\alpha_{i-1}-\alpha_{i}\right) S_{i} y_{n},
\end{array}\right.
$$

where $\epsilon \in\left(0, \frac{1}{\mathrm{~L}}\right)$, $\mathrm{L}$ is the spectral radius of the operator $\mathrm{A}^{*} \mathrm{~A}$ and $\mathrm{A}^{*}$ is the adjoint of $\mathrm{A}$. Suppose that $\alpha_{0}=1$ and $\left\{\alpha_{n}\right\}_{n=1}^{\infty}$ is a strictly decreasing sequence in $[0,1],\left\{\beta_{n, j}\right\}_{n=1}^{\infty} \subset[0,1]$, for $j=0,1,2, \cdots, m$ and the following conditions are satisfied:

(i) $\lim _{n \rightarrow \infty} \alpha_{n}=0, \sum_{n=1}^{\infty} \alpha_{n}=\infty$ and $\sum_{n=1}^{\infty}\left|\alpha_{n-1}-\alpha_{n}\right|<\infty$;

(ii) $\sum_{j=0}^{m} \beta_{n, j}=1, \liminf _{n \rightarrow \infty} \beta_{n, j}>0$ and $\sum_{n=1}^{\infty}\left|\beta_{n, j}-\beta_{n-1, j}\right|<\infty$, for all $j=0,1,2, \cdots$, m. 
Then the sequence $\left\{x_{n}\right\}$ generated by (3.18) converges strongly to $q \in \Omega$, which is the minimum norm solution of the system (1.1).

Proof. Setting $f(x)=0$, the ergodic-type iterative method (1.5) is equivalent to (3.18). By Theorem 3.1, we obtain that

$$
\langle-\mathrm{q}, w-\mathrm{q}\rangle \leqslant 0, \quad \forall w \in \Omega
$$

Therefore,

$$
\|\mathrm{q}\|^{2} \leqslant\langle\mathrm{q}, w\rangle \leqslant\|\mathrm{q}\|\|w\|, \quad \forall w \in \Omega,
$$

which implies that $\|\mathrm{q}\| \leqslant\|w\|$, for all $w \in \Omega$. That is, $\mathrm{q}$ is the minimum norm solution of the system (1.1). This completes the proof.

Theorem 3.3. Let $\mathrm{H}_{1}$ and $\mathrm{H}_{2}$ be two real Hilbert spaces. Let $\mathrm{B}: \mathrm{H}_{1} \rightarrow 2^{\mathrm{H}_{1}}, \mathrm{~B}_{\mathrm{j}}: \mathrm{H}_{2} \rightarrow 2^{\mathrm{H}_{2}}$ be some maximal monotone mappings and $A_{j}: H_{1} \rightarrow \mathrm{H}_{2}$ be a family of bounded linear operators for $j=1,2, \cdots, m$. Let $S$ be a nonexpansive mappings on $\mathrm{H}_{1}$ such that $\Omega=\operatorname{Fix}(S) \bigcap \mathscr{S} \neq \emptyset$. For given $\mathrm{u}, \mathrm{x}_{1} \in \mathrm{H}_{1}$ and $\lambda \in(0,1)$, define $\left\{x_{n}\right\}$ in the following manner:

$$
\left\{\begin{array}{l}
u_{n, j}=J_{\lambda}^{B}\left[x_{n}-\epsilon A_{j}^{*}\left(I-J_{\lambda}^{B_{j}}\right) A_{j} x_{n}\right], j=1,2, \cdots, m, \\
y_{n}=\beta_{n, 0} x_{n}+\sum_{j=1}^{m} \beta_{n, j} u_{n, j}, \\
x_{n+1}=\alpha_{n} u+\left(1-\alpha_{n}\right) S y_{n},
\end{array}\right.
$$

where $\epsilon \in\left(0, \frac{1}{L}\right), L$ is the spectral radius of the operator $A^{*} A$ and $A^{*}$ is the adjoint of $A$. Suppose $\left\{\alpha_{n}\right\},\left\{\beta_{n, j}\right\} \subset$ $[0,1]$, for $j=0,1,2, \cdots, m$ and the following conditions are satisfied:

(i) $\lim _{n \rightarrow \infty} \alpha_{n}=0$ and $\sum_{n=1}^{\infty} \alpha_{n}=\infty$;

(ii) $\sum_{j=0}^{m} \beta_{n, j}=1, \liminf _{n \rightarrow \infty} \beta_{n, j}>0$ and $\sum_{n=1}^{\infty}\left|\beta_{n, j}-\beta_{n-1, j}\right|<\infty$, for all $j=0,1,2, \cdots$, m.

Then the sequence $\left\{x_{n}\right\}$ generated by (3.19) converges strongly to $q=P_{\Omega} u$.

Proof. Setting $f(x)=u$ and $S_{i}=S$, the modified iterative method (1.5) is equivalent to (3.19). Then the desired conclusion follows immediately from Theorem 3.1. This completes the proof.

\section{Numerical examples}

In this section, we give a numerical example to illustrate the operational reliability and strong convergence of the ergodic-type algorithm in Theorems 3.1 and 3.2 as follows.

Example 4.1. Let $\mathrm{H}_{1}=\mathrm{H}_{2}=\mathbb{R}^{2}, \mathrm{~A}_{j} \in \mathbb{R}^{2 \times 2}$ be a non-singular matrix operator with spectral radius $L_{j}=\left\|A_{j}^{*} A_{j}\right\|_{2}$, where $A_{j}^{*}$ is an adjoint of $A_{j}$ and $\|\cdot\|_{2}$ is the matrix 2-norm for $j=1,2$. Let $B, B_{1}$ and $B_{2}$ be matrix operators defined by $B=\left(\begin{array}{ll}8 & 0 \\ 0 & 2\end{array}\right), B_{1}=\left(\begin{array}{ll}3 & 0 \\ 0 & 6\end{array}\right)$ and $B_{2}=\left(\begin{array}{ll}4 & 0 \\ 0 & 5\end{array}\right)$, respectively. Since $B, B_{1}$ and $B_{2}$ are positive linear operators and hence maximal monotone, the resolvent operators $J_{\lambda}^{B}=(I+\lambda B)^{-1}$ and $\mathrm{J}_{\lambda}^{\mathrm{B}_{\mathrm{j}}}=\left(\mathrm{I}+\lambda \mathrm{B}_{\mathrm{j}}\right)^{-1}$ are well-defined on $\mathbb{R}^{2}$.

Algorithm 4.2. Put $\alpha_{n}=\frac{1}{2 n}, \beta_{n, j}=\frac{1}{3}, \lambda=\frac{1}{2}$ and $\epsilon=\frac{1}{2}$. Also, $f: \mathbb{R}^{2} \rightarrow \mathbb{R}^{2}$ is defined by $f(x)=\frac{1}{2} x$ and mapping sequences $\left\{S_{n}\right\}_{n=1}^{\infty}: \mathbb{R}^{2} \rightarrow \mathbb{R}^{2}$ are defined by $S_{n}(x)=\frac{n}{n+1} x$. For a given $x_{1}=\left(x_{1}^{(1)}, x_{1}^{(2)}\right)$, compute sequence $\left\{x_{n}\right\}$ in the following way:

$$
\left\{\begin{array}{l}
u_{n, j}=J_{\lambda}^{B}\left[x_{n}-\epsilon A_{j}^{*}\left(I-J_{\lambda}^{B_{j}}\right) A_{j} x_{n}\right], j=1,2, \\
y_{n}=\frac{1}{3} x_{n}+\frac{1}{3}\left(u_{n, 1}+u_{n, 2}\right), \\
x_{n+1}=\frac{1}{2 n} f\left(x_{n}\right)+\frac{1}{2} S_{1} y_{n}+\sum_{i=2}^{n} \frac{1}{2 i(i-1)} S_{i} y_{n} .
\end{array}\right.
$$


Setting $\left\|x_{n}-x^{*}\right\|<10^{-6}$ as stop criterion, then we obtain the following numerical results of scheme (4.1) with some different initial points $x_{1}=x_{1 i}=\left(x_{1 i}^{(1)}, x_{1 i}^{(2)}\right), i=1,2,3$ in Table 1 .

Table 1: Numerical results of (4.1) for different initial points $x_{1}=\left(x_{1}^{(1)}, x_{1}^{(2)}\right)$.

\begin{tabular}{ccccccc}
\hline Iter. $(\mathrm{n})$ & $x_{\mathrm{n} 1}^{(1)}$ & $x_{\mathrm{n} 1}^{(2)}$ & $\chi_{\mathrm{n} 2}^{(1)}$ & $\chi_{\mathrm{n} 2}^{(2)}$ & $x_{\mathrm{n} 3}^{(1)}$ & $\chi_{\mathrm{n} 3}^{(2)}$ \\
\hline 0 & 3.000000 & 5.000000 & -325.000000 & 1427.00000 & -172.800000 & -52.400000 \\
1 & 1.068333 & 1.930804 & -115.736111 & 551.051339 & -61.536000 & -20.234821 \\
2 & 0.322478 & 0.679516 & -34.935159 & 193.933991 & -18.574756 & -7.121332 \\
3 & 0.092459 & 0.233963 & -10.016363 & 66.773031 & -5.325623 & -2.451932 \\
4 & 0.025891 & 0.079929 & -2.804860 & 22.811635 & -1.491322 & -0.837652 \\
5 & 0.007156 & 0.027214 & -0.775180 & 7.766819 & -0.412157 & -0.285201 \\
6 & 0.001961 & 0.009251 & -0.212477 & 2.640124 & -0.112973 & -0.096946 \\
7 & 0.000535 & 0.003142 & -0.057915 & 0.896703 & -0.030793 & -0.032927 \\
$\ldots$ & $\ldots \ldots$. & $\ldots \ldots$. & $\ldots \ldots$. & $\ldots \ldots$. & $\ldots \ldots$. & $\ldots \ldots$. \\
12 & 0.000001 & 0.000014 & -0.000083 & 0.004040 & -0.000044 & -0.000148 \\
15 & 0.000000 & 0.000001 & -0.000002 & 0.000158 & -0.000001 & -0.000006 \\
18 & 0.000000 & 0.000000 & -0.000000 & 0.000006 & -0.000000 & -0.000000 \\
20 & 0.000000 & 0.000000 & -0.000000 & 0.000001 & -0.000000 & -0.000000 \\
\hline
\end{tabular}

Algorithm 4.3. Put $\alpha_{n}=\frac{1}{2 n}, \beta_{n, 1}=\beta_{n, 2}=\frac{2}{5}$ and $\lambda=\frac{1}{4}$. Also, $f: \mathbb{R}^{2} \rightarrow \mathbb{R}^{2}$ is defined by $f(x)=0$ and mapping sequences $\left\{S_{n}\right\}_{n=1}^{\infty}: \mathbb{R}^{2} \rightarrow \mathbb{R}^{2}$ are defined by $S_{n}(x)=\frac{n}{n+1} \chi$. For a given $x_{1}=(3,5)$, compute sequence $\left\{x_{n}\right\}$ in the following way:

$$
\left\{\begin{array}{l}
u_{n, j}=J_{\lambda}^{B}\left[x_{n}-\epsilon A_{j}^{*}\left(I-J_{\lambda}^{B_{j}}\right) A_{j} x_{n}\right], j=1,2, \\
y_{n}=\frac{1}{5} x_{n}+\frac{2}{5}\left(u_{n, 1}+u_{n, 2}\right) \\
x_{n+1}=\frac{1}{2} S_{1} y_{n}+\sum_{i=2}^{n} \frac{1}{2 i(i-1)} S_{i} y_{n} .
\end{array}\right.
$$

Setting $\left\|x_{n}-x^{*}\right\|<10^{-6}$ as stop criterion, then we obtain the following numerical results of scheme (4.2) for $x_{1}=x_{1 \epsilon}$ with different coefficients $\epsilon=0.1,0.5,0.8$ in Table 2 .

Table 2: Minimum norm solution of (4.2) with different coefficients $\epsilon=0.1,0.5,0.8$.

\begin{tabular}{|c|c|c|c|}
\hline Iter.(n) & $\left(x_{n 0.1}^{(1)}, x_{n 0.1}^{(2)}\right)$ & $\left(x_{n 0.5}^{(1)}, x_{n 0.5}^{(2)}\right)$ & $\left(x_{n 0.8}^{(1)}, x_{n 0.8}^{(2)}\right)$ \\
\hline 0 & $(3.000000,5.000000)$ & $(3.000000,5.000000)$ & $(3.000000,5.000000)$ \\
\hline 1 & $(0.340714,0.878148)$ & $(0.303571,0.724074)$ & $(0.275714,0.608519)$ \\
\hline 2 & $(0.064492,0.257048)$ & $(0.051198,0.174761)$ & $(0.042232,0.123432)$ \\
\hline 3 & $(0.014039,0.086528)$ & $(0.009930,0.048507)$ & $(0.007439,0.028792)$ \\
\hline 4 & $(0.003268,0.031154)$ & $(0.002060,0.014400)$ & $(0.001402,0.007183)$ \\
\hline 5 & $(0.000792,0.011673)$ & $(0.000445,0.004449)$ & $(0.000275,0.001865)$ \\
\hline 6 & $(0.000197,0.004491)$ & $(0.000099,0.001411)$ & $(0.000055,0.000497)$ \\
\hline$\ldots$ & ...... & ...... & $\ldots \ldots$ \\
\hline 10 & $(0.000001,0.000114)$ & $(0.000000,0.000017)$ & $(0.000000,0.000003)$ \\
\hline 12 & $(0.000000,0.000019)$ & $(0.000000,0.000002)$ & $(0.000000,0.000000)$ \\
\hline 15 & $(0.000000,0.000001)$ & $(0.000000,0.000000)$ & $(0.000000,0.000000)$ \\
\hline
\end{tabular}


We display the ergodic-type iterative process with three different initial points $x_{1} \in \mathbb{R}^{2}$ in Table 1 . The numerical example shows that the given point $x_{1}$ has a little effect on iteration and Algorithm 4.2 is good in strong convergence and operational reliability. Moreover, based on a same initial point $x_{1}=(3,5)$, we compare the minimum norm solution of the system (1.1) and the fixed point of a countable family of nonexpansive mappings with different coefficients $\epsilon=0.1,0.5,0.8$ in Table 2, which implies that the increasing of $\epsilon$ has an effect on the number of iteration, that is sequence $\left\{x_{n}\right\}$ generated by (4.2) will converge faster to a common solution when $\epsilon$ is increased.

The computations are performed by Matlab R2012a running on a PC Desktop Intel(R) Core(TM)i32330M CPU @2.20GHz 790MHz 1.83GB, 2GB RAM.

\section{Acknowledgment}

The first author was supported in part by the National Natural Science Foundation of China (11471059), Basic and Advanced Research Project of Chongqing (cstc2016jcyjA0101), and Science and Technology Research Project of Chongqing Municipal Education Commission (KJ1500623, KJ1500634).

\section{References}

[1] H. H. Bauschke, P. L. Combettes, Convex analysis and monotone operator theory in Hilbert spaces, With a foreword by Hédy Attouch, CMS Books in Mathematics/Ouvrages de Mathématiques de la SMC, Springer, New York, (2011). 2.5

[2] C. Byrne, Iterative oblique projection onto convex sets and the split feasibility problem, Inverse Problems, 18 (2002), 441-453. 1

[3] C. Byrne, Y. Censor, A. Gibali, S. Reich, The split common null point problem, J. Nonlinear Convex Anal., 13 (2012), 759-775. 1

[4] L.-C. Ceng, Q. H. Ansari, J.-C. Yao, Relaxed extragradient methods for finding minimum-norm solutions of the split feasibility problem, Nonlinear Anal., 75 (2012), 2116-2125. 1

[5] Y. Censor, T. Bortfeld, B. Martin, A. Trofimov, A unified approach for inversion problems in intensity-modulated radiation therapy, Phys. Med. Biol., 51 (2006), 2353-2365.

[6] Y. Censor, A. Gibali, S. Reich, Algorithms for the split variational inequality problem, Numer. Algorithms, 59 (2012), 301-323.

[7] P. L. Combettes, The convex feasibility problem in image recovery, Adv. Imag. Elect. Phys., 95 (1996), 155-270.

[8] J. U. Jeong, Generalized viscosity approximation methods for mixed equilibrium problems and fixed point problems, Appl. Math. Comput., 283 (2016), 168-180. 1

[9] K. R. Kazmi, S. H. Rizvi, An iterative method for split variational inclusion problem and fixed point problem for a nonexpansive mapping, Optim. Lett., 8 (2014), 1113-1124. 1, 1, 2.2, 3

[10] A. Moudafi, Split monotone variational inclusions, J. Optim. Theory Appl., 150 (2011), 275-283. 1, 3

[11] X.-L. Qin, S. Y. Cho, S. M. Kang, Iterative algorithms for variational inequality and equilibrium problems with applications, J. Global Optim., 48 (2010), 423-445. 1

[12] T. Shimizu, W. Takahashi, Strong convergence to common fixed points of families of nonexpansive mappings, J. Math. Anal. Appl., 211 (1997), 71-83. 1, 1

[13] K. Sitthithakerngkiet, J. Deepho, P. Kumam, A hybrid viscosity algorithm via modify the hybrid steepest descent method for solving the split variational inclusion in image reconstruction and fixed point problems, Appl. Math. Comput., 250 (2015), 986-1001. 1, 1

[14] W. Takahashi, Nonlinear functional analysis, Fixed point theory and its applications, Yokohama Publishers, Yokohama, (2000). 2.3

[15] S.-H. Wang, X.-Y. Gong, A. A. N. Abdou, Y. J. Cho, Iterative algorithm for a family of split equilibrium problems and fixed point problems in Hilbert spaces with applications, Fixed Point Theory Appl., 2016 (2016), 22 pages. 1, 1

[16] D.-J. Wen, Y.-A. Chen, Iterative methods for split variational inclusion and fixed point problem of nonexpansive semigroup in Hilbert spaces, J. Inequal. Appl., 2015 (2015), 14 pages. 1, 1

[17] H.-K. Xu, Iterative algorithms for nonlinear operators, J. London Math. Soc., 66 (2002), 240-256. 2.6

[18] L. Yang, F.-H. Zhao, J. K. Kim, Hybrid projection method for generalized mixed equilibrium problem and fixed point problem of infinite family of asymptotically quasi- $\phi$-nonexpansive mappings in Banach spaces, Appl. Math. Comput., 218 (2012), 6072-6082. 2.4

[19] Y.-H. Yao, R. P. Agarwal, M. Postolache, Y.-C. Liou, Algorithms with strong convergence for the split common solution of the feasibility problem and fixed point problem, Fixed Point Theory Appl., 2014 (2014), 14 pages. 1 